\title{
«Lebendiges! Was nützt der tote Kram!» Büchner im Sprachunterricht Deutsch als Fremdsprache
}

\author{
Doris Beyrich \\ Goethe-Institut Madrid
}

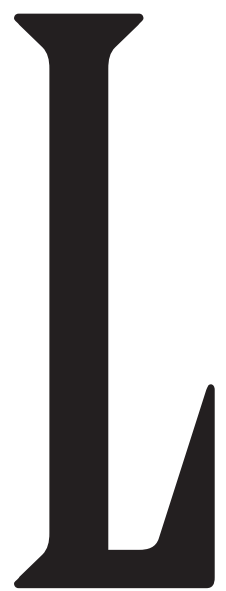

ebendiges! Was nützt der tote Kram!» Diesen Satz malte Büchner in großen Buchstaben in sein Schulheft, das zur Nachschrift über die ,Letternkunst der Alten' gedacht war. Er zeigte kein besonderes Herz für Grammatik und Stillehre, schreibt nur die Überschriften ab und füllt den «toten Kram» mit Volksliedern, die ihm «das Lebendige» verkörpern und übt Kritik an der weltfremden Methode des Unterrichtssystems: «O Herr Doktor! Was sind Verstand, Scharfsinn und gesunde Vernunft? Leere Namen! - Ein Düngerhaufen toter Gelehrsamkeit - dies ist das allein würdige Ziel menschlichen Strebens!» (cfr. Johann 1982: 14).

\section{Wozu Büchner im fremdsprachlichen Deutschunterricht?}

Gerade weil der DaF-Unterricht gewöhnlicherweise das intellektuelle Angebot auf die Alltagssprache reduziert und Teilnehmern (TN) mit einem hohen intellektuellen Niveau und großem kulturellen Wissen auf Grund noch mangelnder Sprachkenntnisse oft das Gefühl vermittelt, intellektuell unterschätzt oder gar kindlich behandelt zu werden, sollte und kann man mit der Literatur als „Gegenmittel « auch den „lebendigen Geist « im Sinne Büchners bei den TN ansprechen. Es geht also darum, das enge Korsett der Alltagssprache und Grammatik des normalen Sprachunterrichts mit lebendigen Inhalten und Formen zu füllen, d.h. mit der Dichtung eine andere Welt zu entdecken, die man ohne sie nicht erhalten kann.

Auch am Goethe-Institut Madrid haben nur Lernende ab dem C1-Niveau in eigens dafür eingerichteten Sonderkursen die Möglichkeit, anspruchsvoller deutscher Literatur überhaupt zu begegnen. Das hat aber zumindest den Vorteil, dass man ein gutes Sprachniveau voraussetzen kann und auch die Zeit vorhanden ist, manche Aspekte zu vertiefen und ins Gespräch zu kommen, trotz der Tatsache, dass die sprachlichen Möglichkeiten nicht immer ausreichen.

Im Folgenden möchte ich eine Anregung geben, wie man mit Hilfe von Literatur zu einem lebendigen Sprach-Austausch unter den TN kommen kann, der weit über das im Sprachunterricht sonst übliche, manchmal etwas forcierte Suchen nach Sprechanlässen hinausgehen kann. Ich spreche dabei von den Erfahrungen, die ich mit einem Projekt über „Büchner - Literatur

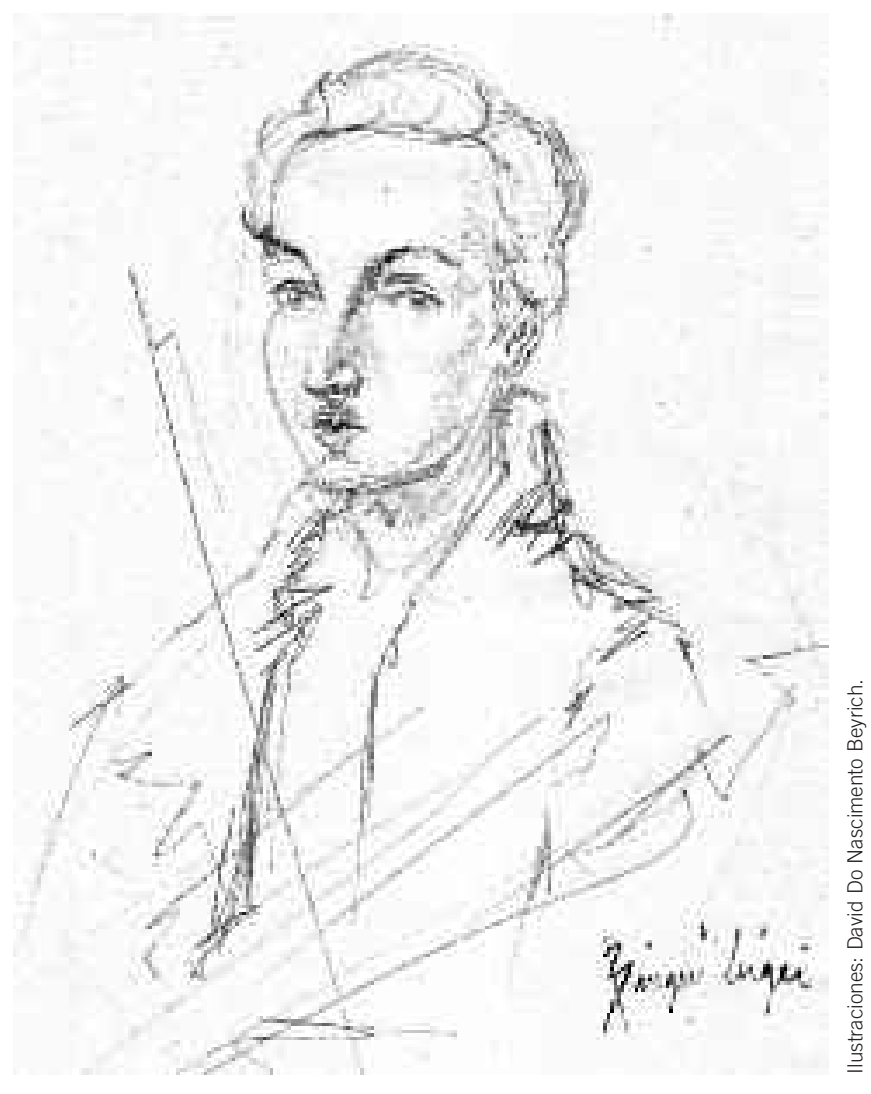

und Politik « in einem Spezialkurs im Jahr 2010 am Goethe-Institut in Madrid machen konnte.

\section{Warum ist Büchner geeignet? Büchners Schreibweise}

Die Suche nach „dem Lebendigen « in der „toten Schrift ( wie kann man tote Strukturen mit Lebendigem füllen?) kann als Leitmotiv von Büchners Schreibweise betrachtet werden. Ich habe sie mir zum Leitfaden für meinen Unterricht gemacht: Einerseits war es mir wichtig, die zugrunde liegende Lebensproblematik Büchners herauszuarbeiten, d.h. Konfliktpotentiale deutlich zu machen, die uns auch heute noch angehen, andererseits wollte ich dennoch den zu einfachen Kurzschluss von biographischen Momenten auf den Sinngehalt des Werks vermeiden. 


\section{Resumen}

El texto expone un proyecto didáctico sobre Georg Büchner en un curso monográfico (nivel MCER C1) Ilevado a cabo por la autora en el curso 2010/11 en el Instituto Goethe de Madrid. Reivindica el trabajo con la literatura reivindicativa del autor del Woyzeck por su fuerza creativa y capacidad ilustrativa de las continuidades de la historia social, política y cultural de Alemania, como la pobreza, la explotación o el conformismo. Propone igualmente el debate sobre las analogías con la situación actual en España y Alemania.

Schlüsselwörter: enseñanza del alemán como lengua extranjera, historia de la literatura alemana, Büchner

\section{Abstract}

Beschrieben wird ein didaktisches Projekt über die Figur Georg Büchners, das die Autorin im Rahmen eines monografischen DaF- Seminars (Niveau C1 GER) im Kurs 2010/11 am Goethe-Institut Madrid entwickelte. Die Arbeit mit den ketzerischen Texten Büchners rechtfertigt sich durch ihre literarische Größe und ihre Fähigkeit, einige Kontinuitäten der deutschen Sozialgeschichte, wie Armut, Ausbeutung und Konformismus darzulegen. Weiterhin wird im Unterricht die Debatte über die Analogien zur gegenwärtigen Situation in Spanien angeregt.

Palabras clave: Deutsch als Fremdsprache, deutsche Literaturgeschichte, Büchner
Es ging also darum, die besondere schriftstellerische Methode Büchners zu erkennen und für den Unterricht zu nutzen. Büchners dichterische Arbeit hat einen ungewöhnlich großen kritisch-empirischen Anteil, der sich sicher dem Wissenschaftler Büchner verdankt. Seinem Schreiben liegen nicht nur historische Quellenstudien zugrunde, sondern er nimmt die historischen Dokumente als Strukturmoment in den Text hinein, dem er dann durch seine große Einfühlungs- und Vorstellungskraft die lebendige Fülle gibt.

So war zum Beispiel die wichtigste Quelle bei Dantons Tod, neben anderen Studien zur Französischen Revolution, Thiers'
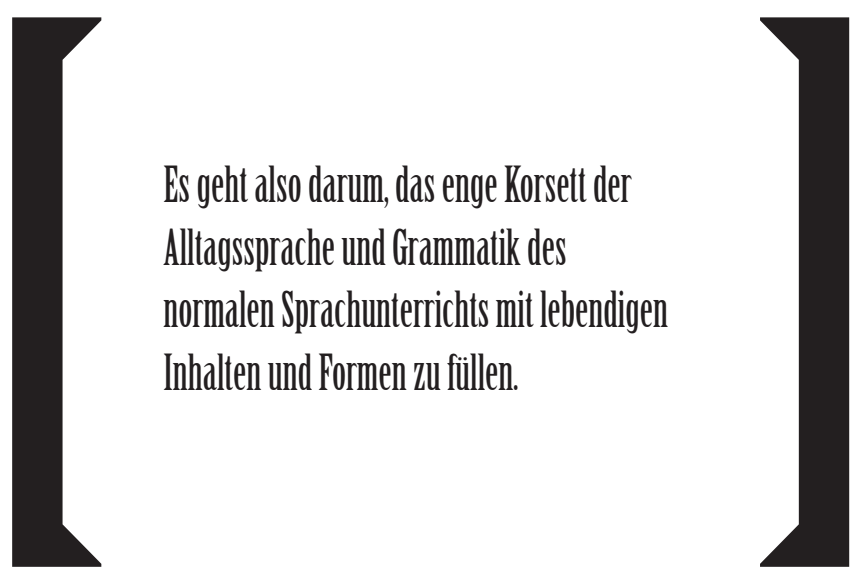

Revolutionsgeschichte (Adolphe Thiers: Histoire de la Révolution francaise, 1823-1827). Hieraus leitete Büchner die szenische Struktur ab und fügte mittels einer damals neuartigen Montagetechnik wörtliche oder leicht abgeänderte Orginalzitate als Redeanteile der Figuren in den Text ein. Dieses Verfahren wiederholt sich auch in den anderen Schriften. Bei Woyzeck sind es Prozessakten und gerichtsmedizinisch/psychiatrische Gutachten, beim Lenz der Bericht des Pfarrers Oberlin, beim Hessischen Landboten demographische und fiskalische Statistiken des Großherzogtums Hessen.

Es bietet sich also geradezu an, «das argumentative Skelett» (Beise 2010: 49) der sachlichen Berichte an geeigneten Stellen mit den von Büchner «literarisch gefüllten Texten» zu vergleichen. Generell scheint mir die Kontrastierung von Texten eine sehr fruchtbare Methode zu sein, unterschiedliche Qualitäten von Li- teratur im Unterricht herauszuarbeiten. Das Prinzip des Gegensatzes setzt die Phantasie und die Denktätigkeit in Gang, stimuliert die Kreativität und die Eigeninitiative der TN und auch ihren «Verstand, Scharfsinn und gesunde Vernunft» (um mit Büchner zu sprechen), wie ich immer wieder feststellen konnte.

\section{Das Spektrum von Büchners Sprache}

Büchners Sprache, die vom derben Volksspa bis zu hoher Poesie reicht, sucht einerseits die "Volksnähe» in Mundarten, Dialekten, Anspielungen auf Volkslieder, Märchen und die Bibel, nährt sich andererseits aber auch von literarischen Verweisen und Bezügen auf reale politische und wissenschaftliche Diskurse aus dem 18. und 19. Jahrhundert. Dieses Spiel zwischen spontaner Volkssprache und hohem diskursivem Niveau, beides aus einer weit zurückliegenden Zeit, stellt einen hohen Anspruch an den Rezipienten und ist auch für Muttersprachler nicht ohne Weiteres verständlich.

Dazu schreibt eine Teilnehmerin:

„Wir wissen, dass Büchner mittels des Theaters das Publikum, das Volk, erreichen wollte. Wofür ist mir nicht so klar. Anscheinend ist es eine allgemeine Meinung, dass Büchner ein Revolutionär war, der für die Vereinigung Deutschlands, das Ende des Absolutismus und die Freiheit und Menschenrechte kämpfte, die Vorurteile des Volks verändern wollte, neue Ideen zu denken geben und einige Wirkung in den armen Herzen der Menschen schaffen wollte. Obwohl diese Ziele selbstverständlich aussehen, sind Büchners Themen in Dantons Tod, zusammen mit seinem Stil oft hoch, klug, sehr scharf und übermäßig kultiviert. War es möglich für das Volk ihn zu verstehen?«

Selbstverständlich gab es die hier angesprochene Verständnisschwierigkeit erst recht für die TN, deren Muttersprache ja nicht Deutsch ist. Deshalb sollte man den TNn empfehlen, die Texte zunächst in der spanischen Übersetzung zu lesen und bei der Lektüre des Orginaltextes Lese- und Interpretationshilfen (Reclam, Klett) zu benutzen.

Die Schwierigkeit von Büchners Sprache erweist sich aber andererseits als ein großer Sprach-Schatz. Die bereits erwähnten reichhaltigen Bezüge zu Volksliedern, Märchen usw. erlauben für den Deutschunterricht eine erste Annäherung, in- 
dem man die Anspielungen Büchners mit ihrem Original konfrontiert. Man kann z. B. die Volkslieder, die sowohl in Dantons Tod als auch im Woyzeck (besonders in der Charakterisierung der Frauen) eine Rolle spielen, als Ganzes vorstellen und fragen, warum Büchner wohl auf dieses Lied Bezug genommen hat. Auf diese Weise können die TN sowohl einen Schatz an Liedern kennenlernen als auch gleichzeitig den Aspekt einer Szene tiefer beleuchten. Hier lassen sich viele Beispiele finden. Für den Woyzeck z.B. ist auch die Gegenüberstellung des Anti-Märchens der Großmutter mit dem Sterntaler-Märchen der Brüder Grimm ein geeigneter Ausgangspunkt.

\section{Die Aktualität Büchners}

Zum Anderen bieten diese Bezüge die unterschiedlichsten Anlässe zu Diskussionen, die bis in unsere Gegenwart reichen. Wie kaum ein anderer Dichter ist Büchner seiner Zeit voraus und berührt uns auch heute noch. Das mag merkwürdigerweise sogar daran liegen, dass er sich nicht als Dichter sah und dennoch eine Dichtung hervorgebracht hat, die - mit den Worten Celans - als Dichtkunst „,den Akut des Heutigen setzt. « (Celan 1972: 91)

Ein Dichter, dem es um Leben und Wirklichkeit geht, nicht um Rhetorik und Stil, der einer Verzweiflung an der Welt zum sprachlichen Ausdruck verhalf und die Suche nach einem Ausweg, nach einem neuen Entwurf provoziert, verlangt also auch einen lebendigen Austausch von Gedanken und Nachdenken. Ein Unterricht, der diese Dringlichkeit seines Anliegens in seiner Sprengkraft didaktisch zudecken würde, würde ihm nicht gerecht.

Büchner hat in seinem kurzen Leben als Dichter, Revolutionär und Wissenschaftler eine so intensive Nachwirkung erreicht, dass sie immer noch andauert: im politischen Kampf um die Menschenrechte, in der Entwicklung des Theaters über den Expressionismus bis heute, für die Moderne Musik (Alban Berg/Zimmermann) und er hat auch die nachfolgenden Schriftsteller in ihren Büchner-Preisreden zu den besten literarischen Selbstzeugnissen inspiriert.

Dieser revolutionäre Keim, der eine ansteckende Wirkung zu eigener Kreativität und Denken auslösen kann, sollte im Kursgespräch erlebbar werden. Deshalb wollte ich dem Lebensimpuls seines dichterischen Schaffens folgen und seinen Lebens- und Denkweg nachzeichnen, der ihn von der Begeisterung und der Hoffnung auf eine Revolte (Hessischer Landbote) zur Enttäuschung an der Massenbewegung und ihrer Führer (Dantons Tod) zum Interesse am Einzelschicksal eines unbedeutenden Individuums, eines «Geringen» als Anti-Helden ( Woyzeck) führte. Es sollte aber auch deutlich werden, dass Büchner trotz allem intellektuellen Pessimismus, die soziale Frage, die als Herausforderung hinter aller Politik steht, nie aufgegeben hat und ihm mit seiner Dichtung, wird man sagen können, «die Trauerarbeit im Schönen» (von Becker 1980) gelang

\section{Vorstellung meines Unterrichts}

Gute Kenntnisse über Literatur und Geschichte konnte ich bei meinem gebildeten Teilnehmerkreis günstigerweise voraussetzen. Daher forderte ich sie auf, ihr Wissen in Form von Referaten in den Unterricht einzubringen. Zu Anfang stellte ich eine Auswahl an Themen bereit, so dass jeder eine Arbeit (auch frei gewählt) übernehmen konnte, die im Laufe des Kurses vorgestellt wurde. Wie die Referate auch gezeigt haben, besaßen die Lerner die Kompetenz, sich einen Gesamtüberblick zu verschaffen und den historisch-philosophischen Hintergrund zu erarbeiten. Meine Rolle beschränkte sich dabei auf die sprachliche Korrektur vor und die dezente Moderation während der Präsentation.

Im Unterricht konfrontierte ich oft konkrete, nicht zu lange Texte oder Textausschnitte mit gegensätzlichen oder ähnlichen Texten anderer Autoren, um den Blick auf die Sprachgestaltung zu schärfen und von daher zu einer Diskussion des Inhalts zu kommen, die auch aktuelle Problematiken aufwirft. Ich habe dabei immer dem Kursgespräch im Anschluss an die Analyse, dem lebendigen Austausch von Gedanken viel Raum gegeben. Dabei ging es sowohl um die Sprache als Mittel lebendiger Kommunikation als auch um die Literatur als Denkanstoß und als Anlass zur Interpretation.

Auch als Sprachlernanlass ziehe ich die Diskussion im Plenum vor. So wird Neugier am Gesagten geweckt, die bei künstlich angelegten Fragestellungen schnell erlahmen kann. Gerade die spontanen Äußerungen im Plenum beleben den Austausch über wichtige Themen, die in ihrer Orginalität in der Gruppenarbeit verloren gingen.

\section{«Friede den Hütten! Krieg den Palästen!»}

Die Zeit als Revolutionär: Der Hessische Landbote (1834)

Diese Schrift, an deren Konzeption auch Büchners Freund und Mitkämpfer Ludwig Weidig beteiligt war, gilt als «die bedeutendste revolutionäre Flugschrift des deutschen Vormärz» (Mai 2001) und brachte Büchner den Ruf ein, der revolutionärste Autor der deutschen Literatur zu sein.

Um den ganz neuen revolutionären Ton Büchners deutlich zu machen, habe ich den Anfang der berühmten Flugschrift mit dem Gedicht „Gebet « (1832) von Eduard Mörike konfrontiert (vgl. Knapp 2000: 88). Es sind sehr sprechende Zeugnisse, zeitnah entstanden, die zwei extrem entgegengesetzte Haltungen zeigen: einerseits den Rückzug in die Innerlichkeit bei Mörike («holdes Bescheiden») und andererseits die revolutionäre Kampfansage gegen die Miss!-Verhältnisse bei Büchner.

Zum Einstieg habe ich zunächst einmal das Mörike-Lied in der Vertonung Hugo Wolfs vorgespielt (1). Die wunderbare Musik, die, um mit Schubert zu sprechen, «in eine bessere Welt entrückt», ließ eine nachdenklich- melancholische Stimmung in der Klasse zurück. In der anschließenden Betrachtung kamen folgende Fragen und Gegenwartsbezüge auf:

- Wie soll man angesichts scheinbar unveränderbarer Verhältnisse reagieren?

- Kann die Kunst ein «Heilmittel» sein für die vielen Enttäuschungen im politischen, alltäglichen (Lebens-)kampf?

- Ist das Verzichtenkönnen nicht notwendig, die Wendung ins Innere?

- Auch heute blühen die unterschiedlichsten spirituellen Angebote neben neuen Formen der politischen Empörung.

Nach dem Besprechen der Wirkung habe ich ein Arbeitsblatt 
mit dem Anfang von Büchners Flugschrift, Worterklärungen und einem Arbeitsauftrag zum Vergleich beider Texte verteilt:

«Friede den Hütten! Krieg den Palästen!

Im Jahr 1834 sieht es aus, als würde die Bibel Lügen gestraft. Es sieht aus, als hätte Gott die Bauern und Handwerker am 5ten Tage, und die Fürsten und Vornehmen am 6ten gemacht, und als hätte der Herr zu diesen gesagt: Herrschet über alles Getier, das auf Erden kriecht, und hätte die Bauern und Bürger zum Gewürm gezählt. Das Leben der Vornehmen ist ein langer Sonntag, sie wohnen in schönen Häusern, sie tragen zierliche Kleider, sie haben feiste Gesichter und reden eine eigne Sprache; das Volk aber liegt vor ihnen wie Dünger auf dem Acker. Der Bauer geht hinter dem Pflug und treibt ihn mit den Ochsen am Pflug, er nimmt das Korn und läßt ihm die Stoppeln. Das Leben des Bauern ist ein langer Werktag; Fremde verzehren seine Äcker vor seinen Augen, sein Leib ist eine Schwiele, sein Schweiß ist das Salz auf dem Tische des Vornehmen.(...)» (Büchner 2006: 7)

Schon das kämpferische Motto der Flugschrift, das aus der Französischen Revolution von 1789 stammt, zeigt, dass es sich hier um einen Aufruf zum Aufstand handelt. Dieser Text ist kein Ge-

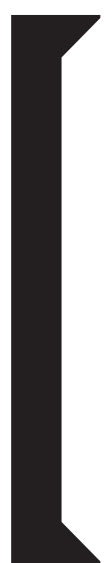

Dieser revolutionäre Keim, der eine ansteckende

Wirkung zu eigener Kreativität und Denken

auslösen kann, sollte im Kursgespräch

erlebbar werden.
- Welche Bedingungen sind nötig, damit so etwas wie ein revolutionärer Impuls auch Breitenwirkung bekommt?

- Warum die politische Apathie in weiten Kreisen der Bevölkerung?

- Zum Zeitpunkt meines Büchnerkurses gab es die 15M- Bewegung noch nicht. Heute könnte man natürlich darüber diskutieren, welche Chancen die Bewegung wohl hat.

"Wenn in unserer Zeit etwas helfen soll, so ist es Gewalt» Um Büchner als Revolutionär vorzustellen, habe ich die Schulfunksendung des Bayerischen Rundfunks «Und dann nehme ich den Schießprügel» zu Hilfe genommen, die die TN zu Hause auch nachhören und nachlesen konnten (2). Für die Aktivierung des Vorwissens und zur Einstimmung auf das Thema eignen sich zwei Dokumente, die als Faksimile präsentiert und einander gegenübergestellt werden können: Der Erstabdruck des Hessischen Landboten mit dem warnenden Vorbericht, der Büchners Mitstreiter Weidig zu verdanken ist, so wie der Steckbrief, mit dem Büchner gesucht wurde. In einer Gruppe mit weniger Vorwissen könnte man über die Zusammenhänge spekulieren und dann die nötigen Informationen durch einen Lehrervortrag ergänzen.

Im Gegensatz zu den Intellektuellen seiner Zeit, wie Heine, Börne und den Burschenschaften, ging es Büchner nicht vordringlich um den Kampf gegen die starken Einschränkungen der Rede- und Pressefreiheit, die drückenden Zensurbestimmungen. Ihm ging es um eine Revolution von «unten», eine Erhebung der Massen zur Beseitigung vor allem der materiellen Not und der sozialen Ungerechtigkeit:

\begin{abstract}
„Die Tendenz der Flugschrift hatte den Zweck, die materiellen Interessen des Volks mit denen der Revolution zu vereinigen, als dem einzig möglichen Weg, die letztere zu bewerkstelligen. - ...Wenigstens sagte er (Büchner) oft, der materielle Druck, unter welchem ein großer Teil Deutschlands liegt, ist ebenso traurig und schimpflich als der geistige; und es sei in seinen Augen bei weitem nicht so betrübend, dass dieser oder jener Liberale seine Gedanken nicht drucken lassen dürfe, als da viele tausend Familien nicht im Stand wären, ihre Kartoffeln zu schmelzen.» (August Becker im Hochverratsprozess, in Enzensberger 1974: 120)
\end{abstract}

Es sollte erstens deutlich werden, dass der revolutionäre Impuls seines Schreibens sowohl aus tiefem Mitgefühl für «die Geringsten», die Hungernden und Unterdrückten und aus gerechtem Zorn und Empörung gegen die bedenkenlosen Machthaber entsprang, als auch auf einer realistischen Beurteilung und fundierten Analyse des gesellschaftlichen status quo beruhte. Die agitatorische Absicht der Flugschrift war der Versuch einer Aufklärung des Volkes, die den Bauern an Hand der Fiskalstatistik vorrechnete und zeigte, „dass sie einem Staate angehören, dessen Lasten sie größtenteils tragen müssen, während andere den Vorteil davon beziehen « (Meyer 1987: 172), und ging davon aus, dass politisch nützliche Veränderungen nur vom Volk selbst bewirkt werden können.

Zweitens, dass die von ihm gegründete Gesellschaft für Menschenrechte in Giessen und Darmstadt (1834) kein Debattier- 
club war, sondern eine illegale Widerstandsgruppe, die ihren politischen Kampf im vollen Risiko der Verhaftung und unter Einsatz ihres Lebens führte.

Die Vorbereitung auf das Hören des Radio-Features habe ich mit der Analyse der Sprache des Landboten verbunden. Zu diesem Zweck habe ich die Original-Zitate Büchners, die in der Sendung vorkommen und andere aussagekräftige Zitate der Flugschrift auf Kärtchen verteilt, von denen die TN jeweils ein Zitat in Partnerarbeit nach literarisch rhetorischen Mitteln analysieren und kommentieren sollten, wie Wortwahl, Metaphorik, Wiederholungen, Übertreibung, starke und kritische Betonung der Gegensätze.

Hier ein paar Beispiele, die sich auch in den regulären DafUnterricht integrieren lassen:

Anspielung auf die Bibel. Um die große Masse des Volkes zu gewinnen, die mit ihrer materiellen Not beschäftigt war, scheut Büchner sich nicht, die Bibelfrömmigkeit seiner Adressaten zu nutzen und das Potential der biblischen Sprache für die aufrührerische Wirkung einzusetzen. Die Anspielung auf die alttestamentarische Schöpfungsgeschichte gleich am Anfang potenziert das Gefühl der Empörung, wenn sozusagen das biblische Versprechen, das jeder ein Teil des Ganzen sei - mit der realen Situation der Ungleichheit der Menschen auf der Erde konfrontiert wird: „Im Jahr 1834 sieht es aus, als würde die Bibel Lügen gestraft...«.

Hyperkonkrete Metaphern. Der bildkräftige Stil steht ganz im Zeichen der Französischen Revolution: «Der Fürst ist der Kopf des Blutegels, der über euch hinkriecht, die Minister seine Zähne und die Beamten sein Schwanz».

Extreme Betonung der Gegensätze. „Das Leben der Vornehmen ist ein langer Sonntag, ...Das Leben des Bauern ist ein langer Werktag; Fremde verzehren seine Äcker...«

Aufforderungscharakter als Strukturmoment und rhetorische Frage.

„Die Justiz ist in Deutschland seit Jahrhunderten die Hure der deutschen Fürsten..(...) Ihr dürft euren Nachbarn verklagen, der euch eine Kartoffel stiehlt; aber klagt einmal über den Diebstahl, der von Staats wegen unter dem Namen von Abgabe und Steuern jeden Tag an eurem Eigentum begangen wird, damit eine Legion unnützer Beamten sich von eurem Schweiße mästen mag: klagt einmal, daß ihr der Willkür einiger Fettwänste überlassen seid und da diese Willkür Gesetz heißt, klagt, daß ihr die Ackergäule des Staates seid, klagt über eure verlornen Menschenrechte: Wo sind Gerichtshöfe, die eure Klage annehmen, wo die Richter, die rechtsprächen? (...)«

Dieses Zitat lässt sich auch als kreativer Schreibanlass einsetzen. Die TN können unter Beibehaltung der Strukturen Büchners einen eigenen aktuellen Klage-Text schreiben.

Vergleich mit Heinrich Heine und Kästner. Um die radikalrevolutionäre Haltung Büchners zu veranschaulichen, die bis zur Legitimierung von Gewalt geht, habe ich noch einen Brief Büchners ergänzend herangezogen und mit zwei anderen politischen Schriftstellern konfrontiert:
"Meine Meinung ist die: Wenn in unserer Zeit etwas helfen soll, so ist es Gewalt. Wir wissen, was wir von unseren Fürsten zu erwarten haben. Alles, was sie bewilligten, wurde ihnen durch die Notwendigkeit abgezwungen. Und selbst das Bewilligte wurde uns hingeworfen, wie eine erbettelte Gnade und ein elendes Kinderspielzeug(...) Man wirft den jungen Leuten den Gebrauch der Gewalt vor. Sind wir denn aber nicht in einem ewigen Gewaltzustand? Weil wir im Kerker geboren und großgezogen sind, merken wir nicht mehr, daß wir im Loch stecken mit angeschmiedeten Händen und Füßen und einem Knebel im Munde». (Brief an die Familie, April 1833, in Büchner 2006: 366).

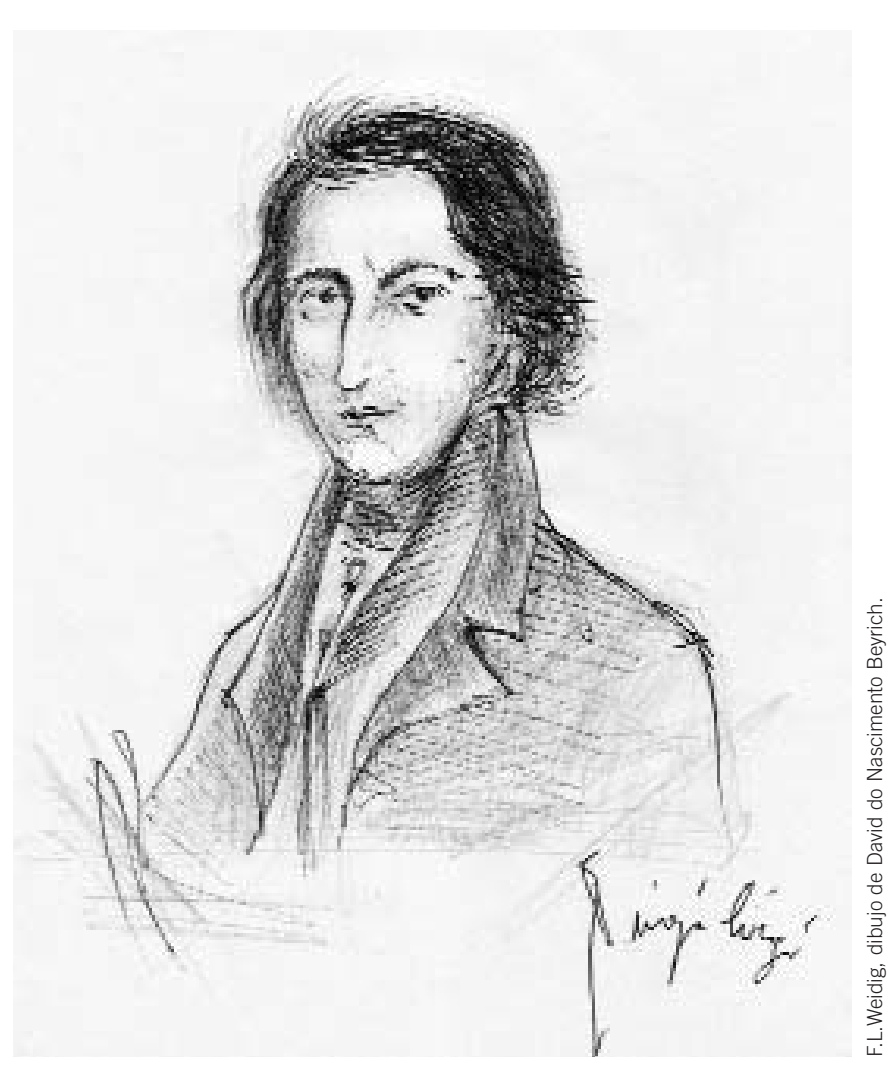

Sein Zeitgenosse Heinrich Heine, der 1831 nach Paris in die Emigration ging, um der politischen Verfolgung und der Zensur zu entgehen und frei schreiben zu können, verfasste dort den Gedichtzyklus Deutschland. Ein Wintermärchen (1843). Er steht den Gedanken des jungen Marx, den er persönlich kennen gelernt hatte, sehr nahe. Aus dem Anfang des Zyklus habe ich die Strophen ausgewählt, mit denen er dem christlichen Entsagungslied sein «besseres Lied» entgegensetzt:

„Ein neues Lied, ein besseres Lied, /O Freunde, will ich euch dichten!

Wir wollen hier auf Erden schon/ Das Himmelreich errichten $\ll(. .$.

Während Heines politische Poesie bei aller Kritik an den ge- 
sellschaftlichen Verhältnissen noch an ein zukünftiges Deutschland glaubt, ist bei Erich Kästner - nach dem 1. Weltkrieg und dem sich anbahnenden Nationalsozialismus - nichts mehr von diesem Optimismus zu spüren. Da wir gerade in der Vorweihnachtszeit waren, habe ich noch zum Vergleich das nicht ganz so christliche, bürgerliche Weihnachtslied: "Morgen, Kinder, wird's was geben» der satirischen Version Erich Kästners gegenüber gestellt: «Weihnachtslied, chemisch gereinigt « (1932):
«Morgen, Kinder, wird's nichts geben!
Nur wer hat, kriegt noch geschenkt.
Mutter schenkte euch das Leben.
Das genügt, wenn man's bedenkt.
Einmal kommt auch eure Zeit.
Morgen ist's noch nicht soweit.
Doch ihr dürft nicht traurig werden.
Reiche haben Armut gern.
Gänsebraten macht Beschwerden.
Puppen sind nicht mehr modern.
Morgen kommt der Weihnachtsmann.
Allerdings nur nebenan.
(...)
Morgen, Kinder, wird's nichts geben!

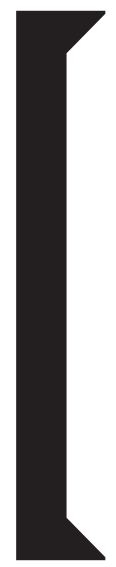

Man wirft den jungen Leuten den

GebrauchderGewalt vor. Sind wirdennabernicht

in einem ewigen Gewaltzustand?

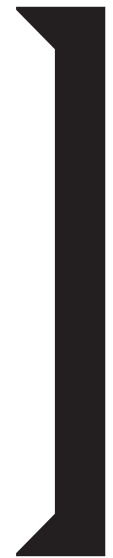

Wer nichts kriegt, der kriegt Geduld!

Morgen, Kinder, lernt fürs Leben!

Gott ist nicht allein dran schuld.

Gottes Güte reicht so weit ...

Ach, du liebe Weihnachtszeit!»

Gleichzeitig habe ich noch ein Zitat aus dem Landboten dazu gegeben, das sich auch als kreativer Schreibanlass anbietet. Die TN können die vorgegebene Struktur nutzen und mit ihren eigenen aufklärerischen Impulsen ergänzen: Was sieht Ihrer Meinung nach die Mehrheit der Leute heute zu wenig?

„...Geht einmal nach Darmstadt und seht, wie die Herren sich für euer Geld dort lustig machen, und erzählt dann euren hungernden Weibern und Kindern, daß ihr Brot an fremden Bäuchen herrlich angeschlagen sei, erzählt ihnen von den schönen Kleidern, die in ihrem Schweiß gefärbt, und von den zierlichen Bändern, die aus den Schwielen ihrer Hände geschnitten sind, erzählt von den stattlichen Häusern, die aus den Knochen des Volks gebaut sind; (....) Das alles duldet ihr, weil euch Schurken sagen: »diese Regierung sei von Gott. « Diese Regierung ist nicht von Gott, sondern vom Vater der Lügen. (...)»

In Konfrontation mit Kästners Text sollten die TN Unterschiede und Gemeinsamkeiten herausarbeiten und überlegen, wie das von Büchner Gemeinte vielleicht einfacher ausgedrückt werden könnte, und dem vehement emotionalisierenden Sprachgestus den eigentlichen Aussagegehalt (die Sachinformation) entnehmen, den Kästner nüchterner ausgedrückt hat: «Gott ist nicht allein dran Schuld». Beiden ist die Sympathie für die Armen gemeinsam, aber Büchner schürt mit seiner Sprache die Empörung mit der Hoffnung auf einen Volksaufstand. Kästner dagegen geht es um die aufrechte Haltung des Einzelnen, der dank seines Wissens um die Verhältnisse seinen Stolz im Abstand des beobachtenden Denkens bewahrt, wie er selbst es auch mit seiner Haltung gezeigt hat, als er zur öffentlichen Verbrennung seiner eigenen Schriften als Zuschauer und Zeuge erschien.

\section{Das Konfliktpotential um eine Wortwahl: «Reiche» oder "Vornehme»}

«Das Verhältnis zwischen Armen und Reichen ist das einzige revolutionäre Element in der Welt» (Brief an Gutzkow, Straßburg, März 1835, in Büchner 2006: 400). Mit dem Ziel, die TN für die Bedingungen der Entstehung von Büchners erstem Drama Dantons Tod (1835) und dem darin gestalteten Konflikt zwischen Danton und Robespierre (Gemäßigte/Radikale) zu sensibilisieren, wollte ich eine Lebensproblematik Büchners herausstellen, die sich in der Zusammenarbeit am Hessischen Landboten mit Ludwig Weidig zeigte. Dazu habe ich den Aufsatz von H.M.Enzensberger, «Politischer Kontext 1834» (Enzensberger 1974: 48-53) als Hausaufgabe zum Lesen aufgegeben, damit die Rolle von Weidig als gleichberechtigt neben Büchner erscheinen kann und die Zusammenarbeit und der Konflikt zwischen ihnen deutlich wird. Hierbei ging es mir auch darum, den weniger bekannten Freund und Mitkämpfer Ludwig Weidig aus Büchners Revolutionszeit vorzustellen und anhand eines konkreten Beispiels ein Konfliktpotential sichtbar zu machen, das in einer akademischen Behandlung des Themas oder auf der Ebene einer Schullektüre eher untergeht.

Im Januar 1834 lernte Büchner den Pfarrer und Schulrektor Friedrich Ludwig Weidig kennen, Kopf der revolutionären republikanischen Bewegung in Hessen. Weidig sollte den Druck des Hessischen Landboten vermitteln, nahm aber eine (die Armutsfrage) entschärfende Umarbeitung des Büchnerschen Manuskripts vor, die Büchner diametral entgegenlief. Von ihm stammt auch der Vorspann des politischen Pamphlets, der von seinen berechtigten Bedenken im Sinne einer realistischen Einschätzung der Gefahren für Autoren und Leser zeugte: 
„Dieses Blatt soll dem hessischen Lande die Wahrheit melden, aber wer die Wahrheit sagt, wird gehenkt, ja sogar der, welcher die Wahrheit liest, wird durch meineidige Richter vielleicht gestraft. Darum haben die, welchen dies Blatt zukommt, folgendes zu beobachten:

Sie müssen das Blatt sorgfältig außerhalb ihres Hauses vor der Polizei verwahren; Sie dürfen es nur an treue Freunde mitteilen; Denen, welche sie nicht trauen wie sich selbst, dürfen sie es nur heimlich hinterlegen; Würde das Blatt dennoch bei Einem gefunden, der es gelesen hat, so muß er gestehen, daß er es eben dem Kreisrat habe bringen wollen;

Wer das Blatt nicht gelesen hat, wenn man es bei ihm findet, der ist natürlich ohne Schuld.«

Besonders aber die Ersetzung des Wortes «die Reichen» durch «die Vornehmen» empörte Büchner - dadurch würde das Potential des Kampfes der Armen gegen die Reichen unzulässig entschärft. Sein Freund August Becker gab in einem späteren Verhör Büchners Reaktion auf die Veränderungen der Schrift durch Weidig zu Protokoll: «Er wollte sie nicht mehr als die seinige anerkennen und sagte, dass er (Weidig) ihm gerade das, worauf er das erste Gewicht gelegt habe und wodurch alles andere gleichsam legitimiert werde, gestrichen habe...» (in Enzensberger 1974: 123).

Nun können folgende Fragen zur Diskussion gegeben werden: Ist die Ersetzung der Wortwahl «Vornehme» statt «Reiche» eine geringfügige Änderung oder würden Sie Büchner Recht geben? Was können die Gründe Weidigs für die Änderungen gewesen sein? Welches Wort hätten Sie genommen - warum? Die Frage deutet ein Dilemma an, das dem ewigen Konflikt zwischen den «Gemäßigten» und den «Radikalen» zugrunde liegt. Sie sollte für den Konflikt sensibilisieren, den Büchner in seinem Drama zwischen Danton und Robespierre gestaltet hat.

Tragischerweise wurde für Weidig die Begegnung mit Büchner zum Verhängnis. Ausgerechnet er als der «Gemäßigtere», fiel im April 1835 der Polizei in die Hände und nahm sich nach 18-monatiger qualvoller Isolationshaft im Gefängnis das Leben - am 23. Februar 1837, vier Tage nach Büchners frühem Tod. Büchner aber, dem «Radikaleren» gelang Anfang März 1835 die Flucht nach Straßburg.

Außerdem war es mir wichtig, auf einen noch heute bestehenden Stein des Anstoßes zu verweisen, der eher von Schriftstellern als von Literaturwissenschaftlern angesprochen wird: denn es geht um Fragen nach unserem Handeln in schwierigen politischen Situationen, die sich jeder auch an sich selbst stellen müsste. Dafür habe ich einen Text-Ausschnitt Hans-Magnus Enzensbergers ergänzend hinzugenommen, der die Grenzen einer nur akademischen Herangehensweise an Büchner aufzeigt:

„Es mutet ein wenig seltsam an, wenn die Gebildeten unter ihren Lesern sie (die Flugschrift) einzig und allein Büchner zuschreiben und Weidig als einen lästigen Pfuscher von ihrem Titelblatt verscheuchen wollen. In diesem Verdikt liegt nicht allein eine literarische
Ungerechtigkeit. Es zeigt auch, wie wenig man in Deutschland vom politischen Widerstand und seinen Bedingungen versteht. Eine illegale Flugschrift braucht nicht nur einen Verfasser, sie muss gedruckt und verbreitet werden. Dazu gehört ein Apparat. Wer diesen Apparat aufbaut, läuft Risiken, er bringt sich in größere Gefahr als der Autor; und zwar umso mehr, je exponierter seine Stellung ist. Der zwanzigjährige Student Georg Büchner konnte, als die Behörden zugriffen, ins Ausland gehen; wir können von Glück sagen, dass ihm die Flucht gelang. Für den Beamten und Familienvater Weidig war an keine Emigration zu denken. Er wusste übrigens, dass sich das Netz der Polizei um ihn zusammenzog; er hat die politische Arbeit dennoch bis zum letzten Augenblick fortgesetzt. Seine Autorschaft am Hessischen Landboten hat er mit seiner Freiheit und mit seinem Leben bezahlt. Nur deutschen Akademikern hat es einfallen können, sie zu schmälern.» (Enzensberger 1974: 51-52)

Auch die Reden der Büchnerpreisträger habe ich gerne mit hinzugenommen, weil ich sie zu den besten literarischen Selbstzeugnissen deutscher Schriftsteller zähle. Sie sind geboren aus der großen Herausforderung, die der Büchnerpreis darstellt nicht nur seiner hohen literarischen Qualität wegen, sondern

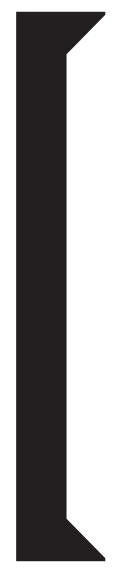

Es geht um Fragen nach unserem Handeln in schwierigen politischen Situationen, die sich jeder auch an sich selbst stellen mïsste.

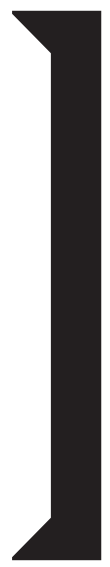

gerade auch deshalb, weil er von der Deutschen Akademie in Darmstadt als eine Art Wiedergutmachung an Büchner gestiftet wurde. In der Büchner-Preis-Rede von Wolfgang Hildesheimer (1966) geht es um die immer noch aktuelle Herausforderung, die Büchner für uns darstellt:

«Die Tradition, alljährlich einen großen Mann zu ehren, der steckbrieflich verfolgt wurde, weil er sich in seinem Land für soziale Gerechtigkeit eingesetzt hatte, der aus der Stadt fliehen musste, in der wir heute seiner gedenken, erfordert Anerkennung. Man muss der deutschen Akademie für Sprache und Dichtung dafür dankbar sein. (...) In der bitteren Erkenntnis, dass in Deutschland Selbstlosigkeit, Gerechtigkeitswille und Integrität nicht zu den Qualifikationen gehö- 
ren, die zur Verwirklichung politischer Ziele beitragen, hat er sich von der Politik zurückgezogen, und wer diesen Rückzug missbilligt, der hat die deutsche Wirklichkeit nicht begriffen. Einen Büchner haben wir heute nicht unter uns, wohl aber genug Georgis - und Schlimmere -, um einen potentiellen Büchner zu verfolgen. (...) Ich habe die letzten Worte nicht in polemischer Absicht gesagt, nicht um einer Dankrede ein Pflichtpensum von Aggressivität gewaltsam anzuhängen; nicht weil sich hier die Gelegenheit bot, sondern weil sie sich aufdrängte, an unumstößliche Wahrheiten zu erinnern,...»

Und er schließt mit der ihm eigenen eleganten Ironie:
„Dennoch hätte die Höflichkeit es mir verboten, dieses Thema bis zu seinem Ende zu behandeln, wüsste ich nicht, dass hier keiner unter uns ist, der nicht gegen jede Obrigkeit für einen Büchner Partei ergreifen würde.»(16)

Zum Schluss noch ein Hinweis

In Büchners erstem Drama, Dantons Tod, das die revolutionäre Legitimierung von Gewalt in Zweifel zieht, geht es nicht nur um den Konflikt zwischen «Gemäßigten» und «Radikalen», auch die Fluchtproblematik (die eigene Haut retten oder bleiben, wo man „hingehört «) und die Frage nach einer möglichen Utopie angesichts des Scheiterns der Revolution sind als Lebensprobleme Büchners in den Text eingegangen.

\section{Anmerkungen}

(1) Link: http://www.youtube.com/watch?v=BtC0d4gakB4 (zum 28.8.2013)

(2) Link: http://www.br.de/radio/bayern2/sendungen/radiowissen/georg-buechner100.html (zum 28.8.2013)
Bibliographie

Beise, Arnd (2010), Einführung in das Werk Büchners. Darmstadt: WBG.

Büchner, Georg (2006), Der hessische Landbote, in Georg Büchner. Schriften, Briefe, Dokumente, Text und Kommentare. Frankfurt: Deutscher Klassiker Verlag. Enzensberger, Hans-Magnus (Hg.)(1974) Georg Büchner/Ludwig Weidig: Der Hessische Landbote, Texte, Briefe, Prozessakten, Frankfurt a.M.: Insel. Celan, Paul (1972), Büchner-Preis-Rede 1960, in Büchner-Preis-Reden 1951-1971, Ditzingen: Reclam.
Johann, Ernst (1982), Büchner. Hamburg: Rowohlt. Knapp, Gerhard P.(2000), Georg Büchner. Stuttgart: Metzler.

Mai, Manfred (2001), Ganz neue Töne, in Geschichte der deutschen Literatur, Weinheim: Beltz.

Mayer, Thomas Michael (Hg.)(1987), Georg Büchner: 1813 - 1837; Revolutionär, Dichter, Wissenschaftler, Katalog der Ausstellung, Mathildenhöhe, Darmstadt: Stroemfeld/Roter Stern.

von Becker, Peter (Hg.)(1980), Georg Büchner. Dantons Tod. Die Trauerarbeit im Schönen. Ein Theater-Lesebuch, Frankfurt a.M.: Schauspiel Frankfurt.

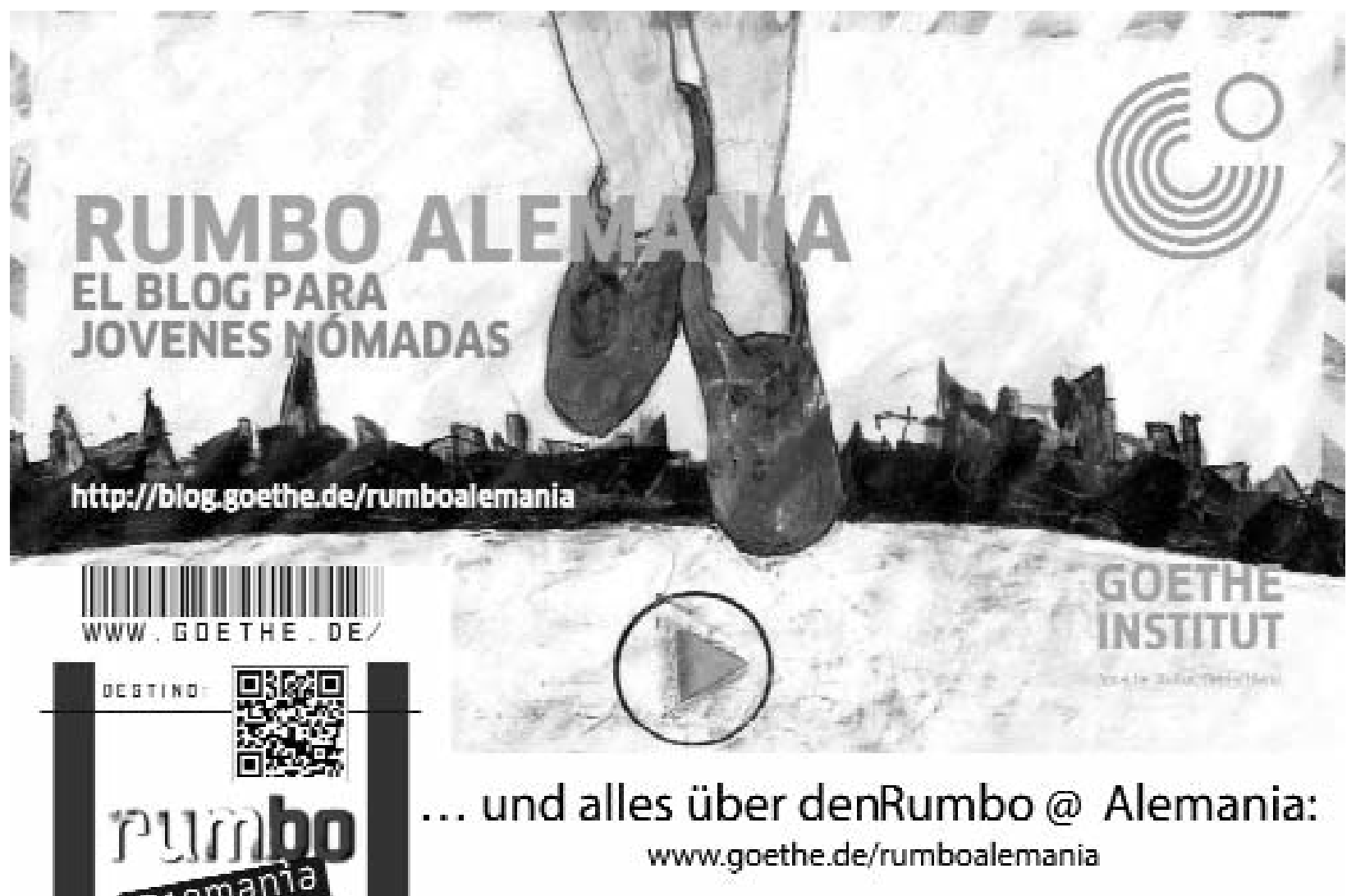

rumbo@facebook >> www.facebook.com/rumboalemania

rumbo@ @witter $\gg$ www.twitter.com/rumboalemania 